\title{
1 A Highly Accurate Ensemble Classifier for the Molecular Diagnosis of ASD at Ages 1 to 4 Years
}

2 Bokan Bao ${ }^{1,2,3,4, *}$, Vahid H. Gazestani ${ }^{1,2^{*}}$, Yaqiong Xiao ${ }^{1}$, Raphael Kim ${ }^{5,6}$, Austin W.T. Chiang ${ }^{1,2}$,

3 Srinivasa Nalabolu ${ }^{1}$, Karen Pierce ${ }^{1}$, Kimberly Robasky ${ }^{6,7,8,9}$, Nathan E. Lewis ${ }^{2,3,4, \$}$, Eric Courchesne Ka $^{1, \$}$

\section{Affiliations}

1. Autism Center of Excellence, Department of Neuroscience, University of California San Diego, La Jolla, CA, USA

Vahid H. Gazestani, Bokan Bao, Yaqiong Xiao, Srinivasa Nalabolu, Karen Pierce \& Eric Courchesne

2. Department of Pediatrics, University of California San Diego, La Jolla, CA, USA Vahid H. Gazestani, Bokan Bao, Austin W.T. Chiang \& Nathan E. Lewis

3. Bioinformatics and Systems Biology Program, University of California San Diego, La Jolla, CA, USA

Bokan Bao \& Nathan E. Lewis

4. Department of Bioengineering, University of California San Diego, La Jolla, CA, USA Bokan Bao \& Nathan E. Lewis

5. Department of Computer Science, University of North Carolina, Chapel Hill, NC, USA Raphael Kim

6. Renaissance Computing Institute, The University of North Carolina at Chapel Hill, Chapel Hill, NC, USA

Raphael Kim \& Kimberly Robasky

7. Department of Genetics, University of North Carolina at Chapel Hill, Chapel Hill, NC 27514, United States

Kimberly Robasky 
medRxiv preprint doi: https://doi.org/10.1101/2021.07.08.21260225; this version posted July 9, 2021. The copyright holder for this preprint (which was not certified by peer review) is the author/funder, who has granted medRxiv a license to display the preprint in perpetuity. It is made available under a CC-BY-NC-ND 4.0 International license.

*These first authors contributed equally: Bokan Bao and Vahid H. Gazestani

$31+$ Corresponding co-equal Senior authors are Nathan E. Lewis and Eric Courchesne

32 Names: Nathan E. Lewis

33 Address: 9500 Gilman Drive MC 0760, La Jolla, CA 92093

34 E-mail: nlewisres@ucsd.edu

35 Name: Eric Courchesne

36 Address: 8110 La Jolla Shores Dr \#201, La Jolla, CA 92037

37 E-mail: ecourchesne1949@gmail.com 


\section{$41 \quad$ Key Points}

42 Question: Since ASD is genetically and clinical heterogeneous, can a single blood-based molecular

43 classifier accurately diagnose ASD at the age of first symptoms?

44

45 Findings: To address heterogeneity, we developed an ASD classifier method testing 42,840 models. An

46 ensemble of 1,076 models using 191 different feature routes and 2,764 gene features, weighted by

47 Bayesian model averaging, demonstrated excellent performance in Discovery and Replication datasets

48 producing ASD classification with the area under the receiver operating characteristic curve (AUC-ROC)

49 scores of $84 \%$ to $88 \%$. Features include genes with immune/inflammation, response to cytokines, 50 transcriptional regulation, mitotic cell cycle, and PI3K-AKT, RAS and Wnt signaling pathways.

51

52 Meaning: An ensemble gene expression ASD classifier has high accuracy across the spectrum of ASD

53 clinical characteristics and across toddlers aged 1 to 4 years.

54 


\section{ABSTRACT}

Importance: ASD diagnosis remains behavior-based and the median age of the first diagnosis remains unchanged at $\sim 52$ months, which is nearly 5 years after its first trimester origin. Long delays between ASD's prenatal onset and eventual diagnosis likely is a missed opportunity. However, accurate and clinically-translatable early-age diagnostic methods do not exist due to ASD genetic and clinical heterogeneity. There is a need for early-age diagnostic biomarkers of ASD that is robust against its heterogeneity.

Objective: To develop a single blood-based molecular classifier that accurately diagnoses ASD at the age of first symptoms.

Design, Setting, and Participants: N=264 ASD, typically developing (TD), and language delayed (LD) toddlers with their clinical, diagnostic, and leukocyte RNA data collected. Datasets included Discovery ( $\mathrm{n}=175$ ASD, TD subjects), Longitudinal (n=33 ASD, TD subjects), and Replication (n=89 ASD, TD, LD subjects). We developed an ensemble of ASD classifiers by testing 42,840 models composed of 3,570 feature selection sets and 12 classification methods. Models were trained on the Discovery dataset with 5fold cross validation. Results were used to construct a Bayesian model averaging-based (BMA) ensemble classifier model that was tested in Discovery and Replication datasets. Data were collected from 2007 to

712012 and analyzed from August 2019 to April 2021.

Main Outcomes and Measures: Primary outcomes were (1) comparisons of the performance of 42,840 classifier models in correctly identifying ASD vs TD and LD in Discovery and Replication datasets; and (2) performance of the ensemble model composed of 1,076 models and weighted by Bayesian model averaging technique.

Results: Of 42,840 models trained in the Discovery dataset, 1,076 averaged AUC-ROC>0.8. These 1,076 models used 191 different feature routes and 2,764 gene features. Using weighted BMA of these features and routes, an ensemble classifier model was constructed which demonstrated excellent performance in 
80 classification accuracy was comparable against LD and TD subjects and in the Longitudinal dataset. ASD

81 toddlers with ensemble scores above and below the ASD ensemble mean had similar diagnostic and

82 psychometric scores, but those below the ASD ensemble mean had more prenatal risk events than TD

83 toddlers. Ensemble features include genes with immune/inflammation, response to cytokines,

84 transcriptional regulation, mitotic cell cycle, and PI3K-AKT, RAS, and Wnt signaling pathways.

85 Conclusions and Relevance: An ensemble ASD molecular classifier has high and replicable accuracy

86 across the spectrum of ASD clinical characteristics and across toddlers aged 1 to 4 years, which has

87 potential for clinical translation. 


\section{INTRODUCTION}

90 ASD is a prenatal ${ }^{1-16}$, highly heritable disorder ${ }^{17}$ that significantly impacts a child's ability to perceive

91 and react to social information ${ }^{18-20}$. Despite this prenatal and strongly genetic beginning, robust and

92 replicable early-age biological ASD diagnostic markers useful at the individual level have not been found.

93 Across the past two decades, ASD diagnosis remains behavior-based and the median age of the first

94 diagnosis remains unchanged at $\sim 52$ months ${ }^{21-24}$, which is nearly 5 years after its first trimester origin.

95 The long delay between ASD's prenatal onset and eventual diagnosis is a missed opportunity. Moreover,

the heterogeneity of ASD genetics and clinical characteristics has imposed barriers to the identification of

97 early-age diagnostic biomarkers that accurately diagnose the broad majority of those with this

98 heterogeneous disorder ${ }^{25}$. Thus, there is a need for early-age diagnostic biomarkers of ASD that can

99 robustly fight against this heterogeneity.

100 Since ASD has a heritability of $81 \%^{17}$, initial attempts focused on genetics as a way towards the

101 discovery of clinically useful biomarkers for precision medicine as well as causal explanations for ASD

102 pathogenesis. While syndromic risk mutations have been described for $>200$ genes in ASD $^{15,26}$, each

103 occurs only rarely in ASD. For $80 \%$ to $90 \%$ of patients, such mutations are not found. Thus, an estimated

$10480 \%$ of ASD individuals are still considered 'idiopathic', wherein little is known about the specific genes

105 and/or environmental factors causing their disorder. In this idiopathic majority of ASD, the risk is likely

106 associated with a large number of inherited common and rare risk variants in each individual child. Studies

107 of polygenic ASD risk found that the combined effect of genetic risk variants in case-control studies

108 accounts for less than $7.5 \%$ of the risk variance ${ }^{27}$; genetic ASD risk scores substantially overlap with

109 controls $^{26}$; and polygenic risk scores are not clinically diagnostic or prognostic at the individual level nor

110 explanatory for the majority of $\mathrm{ASD}^{28}$. Thus, DNA-based mutations or polygenic risk scores may not be

111 useful for the many idiopathic ASD subjects at the translational level. 
RNA biomarkers have been sought using blood gene expression in more than 35 ASD studies since 2006, but the vast majority have been underpowered, older-aged, clinically heterogeneous, and absent

114 replication datasets ${ }^{29-39}$. Some early genetics researchers rejected blood-based biomarkers believing that 115 ASD-relevant dysregulated gene expression must be restricted to the brain. Recent ASD genetics have 116 turned this view on its head: The earliest prenatal drivers of deviant ASD development are, in fact, broadly 117 expressed regulatory genes that are active in blood leukocytes as well as other organs including the 118 prenatal brain ${ }^{1-3,29,30,33,34,39-41}$. Broadly expressed genes that constitute the majority of ASD risk genes are 119 upregulated in the early prenatal life and impact multiple stages of prenatal brain development from the 120 first- and second-trimester proliferation and neurogenesis to neurite outgrowth and synaptogenesis in the 121 third trimester. They do so by disrupting gene expression in signaling pathways such as PI3K-AKT, RAS122 ERK, and Wnt signaling pathways ${ }^{42}$, which, in turn, disrupt prenatal functions.

123 In idiopathic ASD toddlers aged 1-4 year olds, leukocyte gene expression in these pathways is 124 significantly dysregulated. At the group level, the degree of dysregulation is correlated with ASD social symptom severity ${ }^{43}$. Broadly expressed genes in leukocytes from ASD toddlers are also associated with 126 hypoactive brain responses to language, dysregulation of ASD and language relevant genes, and poor 127 language outcomes ${ }^{30}$. Thus, leukocyte gene expression holds the potential for the objective identification 128 of biological subtypes of ASD. In analyses of leukocyte gene co-expression, ASD-associated module 129 eigengene values were significantly correlated with abnormal early brain growth and enriched in genes 130 related to cell cycle, translation, and immune networks and pathways. These same gene sets are very 131 accurate classifiers of ASD vs control toddlers ${ }^{30}$. Meta-analyse or mega-analyse of the wider ASD blood 132 gene expression literature and individual studies ${ }^{29-39}$ find that there appears to be dysregulated gene 133 expression in PI3K-AKT-mTOR, RAS signaling pathways, increased ribosomal translation signal, and 
dysregulation of genes involved in cell cycle, inflammation-related processes, interferon signaling, and the KEGG natural killer cytotoxicity pathway. understanding aspects of ASD cell biology, including those that could be ASD-relevant, ASD-specific, robust, and diagnostic or prognostic. However, if the clinical translational potential of leukocyte transcriptomics is to lead to robust and rigorous classifiers, then high standards for verifying such classifiers should be implemented.

Here we developed, operationalized, and tested a rigorous analytic pipeline to identify molecular diagnostic classifiers for ASD using leukocyte gene expression. Using this platform on leukocyte transcriptomic data from male ASD toddlers at ages 1-4 years old, typically developing (TD), and

144 language delayed (LD) subjects, we systematically analyzed the classification performance of 42,840 145 different models composed of 3,570 different feature selection sets and 12 commonly-used classification 146 methods (Figure 1A). In this way, we developed a highly accurate ensemble diagnostic classifier of male 147 ASD toddlers.

\section{RESULTS}

\section{Development of a robust transcriptomic classifier platform with diverse feature engineering and}

152 To identify potential blood transcriptome biomarkers in our Discovery sample of 175 ASD and TD

153 toddlers (Table 1), we developed a platform that robustly reveals the classification power of blood 154 transcriptome by systematically exploring the performance of 42,840 possible models composed of 3570 155 different feature selection routes followed by 12 classification methods (see Methods, Figure 1A). As 156 shown in Figure 1A, the platform started with removing genes with low variation across samples. Next, 
157 features that differentiate between ASD and TD subjects at expression or co-expression levels were 158 selected using a suite of 102 feature selection methods. Third, to avoid overfitting, we reduced the number 159 of features by collapsing expression data from the correlating genes. Finally, we trained 12 different 160 classifiers based on each of the selected feature sets. To robustly evaluate the performance of each of the 1613570 feature selection routes and the 12 classification methods, we iterated the process 5 times while 162 holding out $20 \%$ of samples and using the remaining $80 \%$ of samples for hyper-parameter selection, 163 feature selection, and classifier training. Thus, each of the 42,840 models started with a "route" that 164 consisted of 1 filtration method, 1 selection method, 1 reduction method, and ended with 1 classification

165

method. The platform reports the average performance of each of the 42,840 models across the 5 held-out folds as measured by area under the receiver operating characteristic curve (AUC-ROC) and area under the precision-recall curve (AUC-PR).

\section{Diverse pipelines successfully classify ASD vs TD}

Since the feature selection methods depended on the characteristics of training transcriptome datasets, some routes were not able to generate features in all five iterations of the platform. Accordingly, the platform successfully classified the data in 15,840 out of 42,840 different ways, including 1320 different routes out of 3570 for feature selection and 12 different classification methods (Supplementary Table 1). From 15,840 trained models, 1822 (11.5\%) models showed classification AUC-ROC of above 0.8 with the max AUC-ROC of 0.856 (Figure 1B, Supplementary Figure 3). Moreover, 1508 of the 1822 models also exhibited an AUC-PR of above 0.8.

These 1822 high-performing models performed well due to their feature routes and were robust to variations in the data or the model. For example, we observed a subset of 175 feature routes (colored with a brown band in Figure 1B) that performed consistently well across different classifiers with a mean 
medRxiv preprint doi: https://doi.org/10.1101/2021.07.08.21260225; this version posted July 9, 2021. The copyright holder for this preprint (which was not certified by peer review) is the author/funder, who has granted medRxiv a license to display the preprint in perpetuity.

It is made available under a CC-BY-NC-ND 4.0 International license .

AUC-ROC score of 0.810 . Additionally, these 1822 high-performing models worked similarly well across all five held-out datasets with a mean range of 0.13 and variance of 0.02 (Supplementary Figure 4). Furthermore, different models that largely overlapped in their feature selection routes also worked well across different classifier methods (Supplementary Figure 3).

To further verify that the performance of these 1822 models was not due to chance alone, we generated five separate randomized datasets by shuffling the sample labels (i.e., ASD or TD) from the Discovery dataset. We next ran the platform on each of the five datasets independently (see Methods). Importantly, the platform identified zero models out of the 1822 with AUC-ROC and AUC-PR of above 0.8 across the five datasets, respectively, suggesting that the high performance of the 1822 models was not due to chance.

\section{Randomized data can be erroneously "classified" at reasonable AUC-ROC levels}

The large number of models that resulted in a high AUC-ROC value were encouraging for the prospect of developing a blood-based classifier to supplement ASD diagnosis. However, depending on the model, a range of AUC-ROC values could be obtained. Thus, the question remained whether this range is significantly different from the AUC-ROC values that one could obtain from trying to classify subjects after randomizing their final diagnosis. To test this, we permuted the sample labels (i.e., ASD and TD) for all subjects in our Discovery set and ran the pipeline to test all feature engineering and classification methods. Importantly, we tested all 42,840 candidate models and found the median AUC-ROC score was 0.5101 with the 95 th $\mathrm{CI}(0.4238-0.6498)$ on the randomized samples. Importantly, we found very rare but erroneous “classification" could be made with AUC-ROC values up to 0.8471 (see Methods, Figure 1D), which exceeded values reported for actual classifiers in the past. Thus, great care must be taken when developing classifiers for ASD to ensure the validity of a classifier. 
Classification is robust to sampling time, validation batches, and distinguishes ASD from language delay subjects

To assess the validity of the top-performing models and their reproducibility, we compared the performance of the 1822 models using two different datasets (i.e., Longitudinal, Replication datasets) that were not part of the discovery dataset nor used in any steps of feature route or classifier optimization(Fig. 1C), as further described below.

209 First, we evaluated the performance of the 1822 high-performing models on a Longitudinal dataset.The

observed high reproducibility of model performance in Longitudinal samples suggests the existence of inherent biological signals in the ASD subjects that are independent of age or sample collection factors

212 (see Methods, eResult 1 in Supplementary).

213 Second, we applied the 1822 models to an independent Replication dataset of 34 ASD and 31 TD. This

214 dataset was assayed separately from the Discovery dataset and was independently analyzed. Notably, of 2151822 models with AUC-ROC above 0.8 in the Discovery dataset, 1076 models (59.0\%; Fisher's Exact 216 Test $P<2.2 \mathrm{e}-16$ ) also had an AUC-ROC above 0.75 for the independent Replication ASD and TD samples 217 (see Methods, eResult 2).

218 Third, we assessed the diagnostic specificity of the models by comparing performance of the 1822 219 models in separating a Diagnostic Specificity dataset of 24 LD toddlers from the Replication dataset. From 2201822 models with AUC-ROC or AUC-PR above 0.8, none (Fisher's Exact Test $P<2.2 \mathrm{e}-16$ ) had an AUC221 ROC or PR-ROC above 0.8 in this LD vs TD dataset. We also assessed the diagnostic specificity of the 222 models by comparing performance of the 1822 models in separating a Diagnostic Specificity dataset of 22324 LD toddlers from the ASD samples. From 1822 models with AUC-ROC or AUC-PR above 0.8, 26 224 (1.42\%; Fisher's Exact Test $P<2.2 \mathrm{e}-16)$ had an AUC-ROC above 0.75 in this LD vs TD dataset (see 225 Methods, eResult 2 in Supplementary). 


\section{Genes driving classification are largely immune-associated}

We characterized the feature routes of 1076 models showing AUC-ROC above 0.8 in the Discovery dataset and above 0.75 in the independent Replication dataset. The 1076 models mostly originated from 191 feature routes. The mean variance of AUC-ROC scores by 12 classifiers on one route is $7.510 \mathrm{e}-04$ $(95 \% \mathrm{CI}$ is $[6.968 \mathrm{e}-04,8.117 \mathrm{e}-04])$.

To identify the biological processes that contribute to these 191 feature routes, we scored all genes in these routes based on the number of times they occurred in different 191 routes (Supplementary Table 2). By comparing the top five hundred genes selected by the routes, we found the genes selected by the feature routes had a larger impact on the model performance than the 12 classifiers. Different routes that largely overlapped in selected feature genes also showed highly correlated behavior across different classifier methods (see Methods, Supplementary Figures 5 and 6). We next calculated the enrichment of biological processes among top performing genes using g:Profiler. In this work, the 500 most common genes (Supplementary Table 2) were enriched in the KEGG pathways of inflammation/immune related disease, including Acute myeloid leukemia (KEGG: hsa05221), Measles infection (KEGG: hsa01524) and other significant pathways such as oxidative phosphorylation (KEGG: hsa04070), Ras signaling pathway (KEGG: has04014) and Wnt signaling pathway (KEGG: hsa04310). Similarly, enriched Gene Ontology terms for biological processes were associated with the down-regulation in blood inflammation/immune cell response, transcriptional gene regulation and response to cytokines, which were consistent with previous studies ${ }^{30,34,36}$ (see Methods, Supplementary Table 3).

\section{High-performing models typically classify subjects similarly}


medRxiv preprint doi: https://doi.org/10.1101/2021.07.08.21260225; this version posted July 9, 2021. The copyright holder for this preprint (which was not certified by peer review) is the author/funder, who has granted medRxiv a license to display the preprint in perpetuity.

It is made available under a CC-BY-NC-ND 4.0 International license .

248 We next leveraged our set of 1076 high-performing models to determine if there were different

249 classification approaches that identify and separate ASD subjects into subgroups. To do so, we clustered 250 the 175 subjects in the Discovery datasets based on classification score similarity. The classification scores 251 provide a probabilistic measure on the certainty of a model on the diagnostic status of each discovery 252 sample. It ranges from 0 to 1 with 0 being the highest certainty in TD status and 1 being the highest 253 certainty in ASD status. The hierarchical clustering analysis found only one ASD group separated from 254 TD subjects (Figure 2A). This shows that the 1076 models, overall, worked similarly in their assignment 255 of classification scores to the subjects; that is, there was no evidence that different models selected 256 different groups of ASD subjects. This was also true in analysis of the independent Replication dataset 257 (Figure 2A).

Bayesian model averaging created a single ensemble classifier from the 1076 models

260 We next employed a Bayesian model averaging technique to assign an ensemble classification score to 261 each subject. Each subject's ensemble score was an average of his classification scores across the 1076 262 high-performing models weighted by the model performance as measured by AUC-ROC. We used this 263 ensemble classifier model to calculate the AUC-ROC and AUC-PR values from the composite of the 1076 264 models. This analysis indicated an AUC-ROC of $84.42 \%$ and $87.85 \%$ for Discovery and Replication 265 datasets, respectively (Figure 2B). We observed a similar performance based on PR curves with AUC266 PR of $84.10 \%$ and $91.25 \%$ for the Discovery and Replication datasets, respectively, which is significantly 267 higher than the baseline we created by using the naive Random Forest model with $72.32 \%$ AUC-ROC 268 (ROC.test $P<0.01^{44}$ ) (see Methods, eResult 3 in supplementary).

269 Given that social attention abnormalities are well known in ASD, we enhanced the ensemble model by 270 incorporating toddlers' gaze fixation on non-social (i.e., geometric) images as measured in an eye-tracking 
271 test known as GeoPref test and used in previous studies ${ }^{45-47}$. The updated model was tested on 132 of 175

272 Discovery dataset subjects and 41 of 65 Replication dataset subjects who had available Geo-Fixation data

273 (e.g., moderate or good data quality, total looking time > 50\%). By directly classifying the subjects who

274 had percent fixation on non-social images $>69 \%$ as ASD (GeoPref-subtype $)^{45-47}$, the model AUC-ROC

275 increased to 88.02\% (AUC-PR 88.10\%) and 89.42\% (AUC-PR 92.11\%) for Discovery and Replication

276 datasets (see Methods, Supplementary Figure 7).

277

ASD subjects with below-ASD-mean score were more likely to have had a prenatal risk event

279 Subsequently, we examined the clinical characteristics of ASD and TD subjects with different ensemble

classifier scores. First, we stratified ASD toddlers by the mean ASD classifier score of 0.714 and grouped

them into the above-the-mean ASD subgroup and the below-the-mean subgroup (Figure 2C). We next understand its power in distinguishing ASD from TD samples (Figure 2C).

The below-the-mean subgroup had no differential expressed (DE) genes compared with the TD group,

while the above-the-mean ASD subgroup had 1162 DE genes. There were 394 DE genes in the first 500

gene features selected by the 191 feature routes and $700 \mathrm{DE}$ genes in the first 1000 gene features

287 demonstrating the ensemble model captured the dysregulation of gene expression pattern in the above-

288 the-mean subgroup (Supplementary Table 2). After performing the DE gene analysis on the above-the-

289 mean ASD subgroup vs TD, we conducted the enrichment analysis on the biological processes GO terms,

290 and the pathway analysis with the KEGG database using g:Profiler ${ }^{48,49}$. In addition to the

291 inflammation/immune response, transcriptional gene regulation, and response to cytokine, we found more

292 gene ontology terms associated with the mitotic cell cycle. The significant pathways included the cell

293 cycle (KEGG:hsa04110), PI3K-AKT (KEGG:hsa04151), RAS signaling pathways (KEGG:hsa04014), 
294 and Wnt signaling pathways (KEGG:hsa04310), which is consistent with our previous finding ${ }^{42}$ 295 (Supplementary Table 3).

296 Diagnostic and psychometric scores were not significantly different between the above-the-mean ASD 297 subgroup and the below-the-mean ASD subgroup (Supplementary Table 4). However, there were 298 differences in prenatal risk factors, wherein ASD toddlers with classifier scores above the ASD mean had 299 significantly fewer prenatal neurodevelopmental risk events, while ASD toddlers below the mean had 300 disproportionately more prenatal risk scores than TD toddlers (Table 2 and 3). We tested if there was a 301 different ratio of severe prenatal events that could potentially impact ASD development between the two 302 transcriptome ASD subgroups. We found a similar rate of prenatal events between TD subjects and above303 the-mean ASD subjects (Odds Ratio: 0.88, Fisher's Exact Test $P=0.84$ ). However, there was a significant 304 enrichment of prenatal events among the below-the-mean ASD subjects compared to TD subjects (Odds 305 Ratio: 2.78; Fisher's Exact Test $P=0.013$ ). As a negative control, prenatal events that are unlikely to 306 affect ASD development were not enriched among ASD subjects with below the ASD mean score. These 307 results suggest the possible existence of different underlying etiological factors between ASD subjects 308 with above vs below the mean ASD classifier score.

309 Overall, while $83(64.5 \%)$ of ASD subjects showed an ensemble classification score of above 0.714 , 310 only $11(9.73 \%)$ of TD subjects reached this score, underscoring the high performance of the ensemble 311 score in separating ASD and TD cases. This indicates higher ensemble diagnostic scores are strongly 312 driven by significantly DE genes in leukocytes that enrich the GO biological processes described above 313 and in Supplementary table 3, while DE genes are less of a classifier factor in ASD subjects with lower 314 classification ensemble scores.

\section{Discussion}


317 Despite its prenatal beginning ${ }^{1-16}$, ASD diagnosis remains behavior-based and the median age of first 318 diagnosis is about 52 months. Partially due to its genetic and clinical heterogeneity, no single diagnostic marker has been found that can accurately and reproducibly diagnose more than a small subset of affected children. Even among those capable of correctly diagnosing small subsets of ASD infants and toddlers,

321 few have proven clinically useful, cost-effect, and/or practical at the ages when early detection and diagnosis are most needed and could be mostly important for the child and family ${ }^{47}$.

We used a unique approach to this dilemma: We addressed ASD genetic and clinical heterogeneity with classifier heterogeneity. That is, since we expected heterogeneity in classifier gene features, we designed a classifier pipeline to train, test, and validate 42,840 models generated from 3,570 gene feature routes and 12 classification methods to correctly identify ASD at ages 1 to 4 years. Then, rather than selecting a single "best" performing model, we used Bayesian model averaging to bring together 1,076 performing accurately across the ASD spectrum from more affected to less affected. individuals. Non-genetic heterogeneity was also detected here insofar as those with ASD classifier scores below the overall ASD mean tended to have more prenatal risk events in their history than those ASD toddlers with above the mean scores. This opens the important potential to utilize these ASD ensemble classifier scores in future research to home in ASD subtypes more likely to be principally driven by genetic 
versus subtypes more likely to be driven by non-genetic or a combination of non-genetic and genetic

341 factors.

342 Our ensemble features include genes with PI3K-AKT, RAS, and Wnt signaling pathways, 343 immune/inflammation, response to cytokines, transcriptional regulation, and mitotic cell cycle. Meta344 analysis, review, and original data papers on ASD blood gene expression ${ }^{29-39}$ generally show that common 345 ground across many studies is dysregulation in PI3K-AKT-mTOR and RAS signaling pathways, 346 immune/inflammation processes, the KEGG natural killer cell pathway, cell cycle, apoptosis, 347 neurogenesis as well as increased ribosomal translation signal. These main signaling pathways, immune 348 and cell cycle correspondences are notable despite the fact that (1) across more than 35 studies diverse 349 blood-based samples were examined (whole blood, lymphoblastoid cell lines, peripheral blood 350 mononuclear cells, or other types of leukocyte capture methods); (2) most studies did not actively account 351 for sex-related, age-related or clinical-symptom heterogeneity as moderating factors; (3) 84\% of them had 352 less than 100 ASD subjects averaging 28 subjects/study; and (4) most studies focused on older ASD 353 children and adults ${ }^{29-39}$.

354 PI3K-AKT, RAS and Wnt signaling pathways may be pivotal to ASD prenatal neural maldevelopment. 355 Recently, in a large sample study, we discovered that ASD toddlers had significant upregulation of PI3K356 AKT, RAS-ERK and Wnt signaling pathways in both leukocytes and iPSC-derived prenatal neural 357 progenitors and neurons ${ }^{42}$. This leukocyte dysregulation in ASD toddlers aged 1-4 years olds correlated 358 with ASD social symptom severity ${ }^{42}$. Moreover, these pathways in leukocytes are downstream targets of 359 regulatory risk ASD genes ${ }^{42}$.

360 Leukocyte gene expression has high potential not only for objective molecular diagnostic identification 361 of ASD at 1 and 2-year-olds of age, but also for understanding ASD genetic and clinical heterogeneity at 362 early ages. For example, leukocyte expression signatures correlate with brain size in ASD ${ }^{29}$, and to 
atypical cortical patterning subtypes ASD toddlers with poor language outcome ${ }^{50}$. Leukocyte expression also relates to hypoactivation response to affective speech in ASD toddlers with poor language outcome ${ }^{51}$. Finally, we found multivariate leukocyte expression signatures can predict trajectories of response to early intervention treatment ${ }^{52}$, which underscores the mechanistic relevance of leukocytes to ASD and clinically

367 important phenomena that can be individualized to specific patients.

In sum, a large amount of literature, meta-analyses, and the robust leukocyte diagnostic discoveries in the present study, all point to the importance of leukocyte cell biology in ASD and show that ASD-relevant ASD development are broadly expressed regulatory genes, and such genes are active not only in the brain but also in leukocytes and other organs ${ }^{1-3,29,30,42,51,53}$. Over $60 \%$ of ASD risk genes are broadly expressed regulatory genes ${ }^{1,3,16}$, and 97 of the top broadly expressed regulatory ASD risk genes express in leukocytes as well as the brain. Thus, broadly expressed genes that constitute the majority of ASD risk genes are upregulated in the early prenatal life, which impact multiple stages of prenatal brain development from $1^{\text {st }}$ PI3K-AKT, RAS-ERK and Wnt signaling pathways ${ }^{1,3,42}$, which further disrupt prenatal functions. ASD leukocyte expression has high relevance to ASD diagnostic, structural, and neural functional phenotypes and clinically different prognostic endpoints ${ }^{50,51}$.

381 Here we developed an innovative high-performing ASD molecular classifier with heterogeneous gene 382 features designed to address ASD genetic and clinical heterogeneity. This high-level performance in ASD 383 male toddlers aged 1 to 4-year-olds opens the possibility of further refining ASD molecular classifiers 384 optimized for ethnicity, age, and sex. The ensemble gene expression ASD classifier reported here is 385 enriched in gene features known to be involved in ASD prenatal and postnatal pathobiology, and as such, 
medRxiv preprint doi: https://doi.org/10.1101/2021.07.08.21260225; this version posted July 9, 2021. The copyright holder for this preprint (which was not certified by peer review) is the author/funder, who has granted medRxiv a license to display the preprint in perpetuity.

386 it succeeds because of this. Thus, it is more than a signal capable of classifying ASD. Instead, it is a signal 387 of the underlying pathobiological bases of the disorder in a majority of affected toddlers and therefore has 388 implications for future research targeting treatment-relevant mechanisms. 
medRxiv preprint doi: https://doi.org/10.1101/2021.07.08.21260225; this version posted July 9, 2021. The copyright holder for this preprint (which was not certified by peer review) is the author/funder, who has granted medRxiv a license to display the preprint in perpetuity.

It is made available under a CC-BY-NC-ND 4.0 International license.

\section{Acknowledgements}

390 This work was supported by NIMH grant no. R01-MH110558 (E.C., N.E.L.), NIMH grant no. R01-

391 MH080134 (K.P.), NIMH grant no. R01-MH104446 (K.P.), an NFAR grant (K.P.), NIMH grant no.

392 P50-MH081755 (E.C.), and generous funding from the Novo Nordisk Foundation through the Center for

393 Biosustainability at the Technical University of Denmark (grant no. NNF10CC1016517 to N.E.L.).

395 Author Contributions

396 V.H.G., B.B., R.K., A.W.T.C, K.P., K.R., E.C. and N.E.L. conceived the project and designed the experiments.

397 Y.X. and S.N. collected the samples, conducted transcriptome assays and managed the data. V.H.G., B.B. and

398 R.K. analyzed the data. V.H.G., B.B., Y.X., R.K., K.P., E.C. and N.E.L. interpreted the results and wrote the 399 manuscript. E.C. and N.E.L. supervised the project.

400

401 Competing interests

402 The authors declare no competing interests.

403

404 Code Availability

405 We provide the code library in R and Python described in this work through Github:

406 https://github.com/LewisLabUCSD/autism_classifier. We provide jupyter notebooks in python and R to 407 generate our figures and analysis. 


\section{METHODS}

410

411 Participants in this study included 264 male toddlers ages 1 to 4 years (Table 1). About $70 \%$ of toddlers were recruited from the general population as young as 12 months using an early screening, detection, and or study coordinator at the time of their child's enrollment. diagnosis strategy called the Get SET Early procedure ${ }^{22}$. Using this approach, toddlers who failed a broadband screen, i.e., the CSBS IT Checklist ${ }^{23}$, at well-baby visits in the general pediatric community settings were referred to our center for a comprehensive evaluation. The remaining subjects were obtained by general community referrals. All toddlers received a battery of standardized psychometric tests by highly experienced Ph.D.-level psychologists, including the Autism Diagnostic Observation Schedule (ADOS; Module T, 1 or 2), the Mullen Scales of Early Learning, and the Vineland Adaptive Behavior Scales. Testing sessions routinely lasted 4 hours in one day or occurred across 2 separate days. Toddlers younger than 36 months upon initial clinical evaluation were followed longitudinally approximately every 9 months until final diagnosis at ages 2-4 years. Most toddlers were tracked and diagnosed using the appropriate module of the ADOS (i.e., ADOS Module-Toddler, Module-1, or Module-2) between 24-49 months, an age when the diagnosis of ASD is relatively stable ${ }^{24} ; 264$ toddlers had their final diagnostic evaluation between 18 to 24 months. 127 toddlers were ASD, 113 TD, 24 LD (Table 1). The 264 toddlers included in the current study were among those in a 1,269 subjects study of early diagnostic stability in $\mathrm{ASD}^{43}$. Research procedures were approved by the Institutional Review Board of the University of California, San Diego. Parents of subjects underwent Informed Consent Procedures with a psychologist 
431 Transcriptome data based on microarrays included previously published samples ${ }^{25}$ and 46 novel samples.

432 For these, blood samples were collected from each of the 264 subjects; longitudinal blood samples were

433 collected from a subset of 33 subjects (Table 1). They were usually taken at the end of clinical evaluation

434 sessions. To monitor health status, the temperature of each toddler was monitored using an ear digital

435 thermometer immediately preceding the blood draw. The blood draw was scheduled for a different day

436 when the temperature was higher than 99 Fahrenheit. Moreover, the blood draw was not taken if a toddler

437 had some illness (e.g., cold or flu), as observed by us or stated by parents. We collected four to six

438 milliliters of blood into ethylenediaminetetraacetic-coated tubes from all toddlers. Blood leukocytes were

439 captured and stabilized by LeukoLOCK filters (Ambion) and were immediately placed in a $-20^{\circ} \mathrm{C}$ freezer.

440 Total RNA was extracted following standard procedures and manufacturer's instructions (Ambion).

\section{Data processing and differential gene expression analysis of the microarray primary dataset}

443 From each blood sample, leukocyte RNA was extracted, and gene expression was assayed using Illumina

444 HT-12 platform. All arrays were scanned with the Illumina BeadArray Reader and read into Illumina

445 GenomeStudio software (version 1.1.1). Raw Illumina probe intensities were converted to expression

446 values using the lumi package ${ }^{54}$. We employed a three-step procedure to filter for probes with reliable

447 expression levels. First, we only retained probes that met the detection $P$ value $<0.05$ cut-off threshold in

448 at least 3 samples. Second, we required probes to have expression levels above the $95^{\text {th }}$ percentile of 449 negative probes in at least $50 \%$ of samples. The probes with detection $P$ value $>0.1$ across all samples were 450 selected as negative probes and their expression levels were pooled together to estimate the $95^{\text {th }}$ percentile

451 expression level. Third, for genes represented by multiple probes, we considered the probe with the highest

452 mean expression level across our dataset, after quantile normalization of the data. These criteria led to the 453 selection of 14,312 coding genes as expressed in our leukocyte transcriptome data, which highly overlaps 
medRxiv preprint doi: https://doi.org/10.1101/2021.07.08.21260225; this version posted July 9, 2021. The copyright holder for this preprint (which was not certified by peer review) is the author/funder, who has granted medRxiv a license to display the preprint in perpetuity. It is made available under a CC-BY-NC-ND 4.0 International license .

454 with the reported estimate of 14,555 protein-coding genes (chosen based on unique Entrez gene IDs) for 455 whole blood by the GTEx consortium ${ }^{55}$.

457 Building the classifier platform on the Discovery set

458 The Discovery dataset included 175 subjects with 14,132 gene features. The pipeline ran five iterations. 459 At the beginning of each iteration, the pipeline held out $20 \%$ of samples and used the remaining $80 \%$ of 460 samples for hyper-parameter selection, feature selection, and classifier training. In the first step, feature 461 filtration, five methods were used (Supplementary Figure 1), including no (no action), cov (remove 50\% 462 feature with the smaller coefficient of variation), var (remove 50\% feature with smaller variance), cov_var 463 (remove 50\% feature with the smaller coefficient of variation and then remove 50\% feature with smaller 464 variance in the rest), varImportance (keep only the $25 \%$ features with the highest variance).

465 The second step, feature selection, includes seven groups of methods (Supplementary Figure 1) with 466102 methods in total. The methods within each group are conceptually similar, but use different 467 approaches. These groups are no (no action), grn ${ }^{56}$ (genetic regulatory network), z-score, selectV $\mathrm{V}^{57}, \mathrm{svm}^{58}$, 468 GSEA $^{59}$, DE-analysis ${ }^{60}$. The Grn group has 14 methods, based on gene regulatory network analysis. The 469 Z-score group has 4 methods for selecting features with high z-score variance. It picks the features with 470 variance in the first quartile and then selects features within the 3rd quartile of the context likelihood of 471 relatedness. The SelectV group has 54 methods and bases on the "Variable Selection for High472 Dimensional Supervised Classification"57. In this group, features are first filtered by 'ExpHC','HC', 'Fair' 473 methods (with/without comvar). The remaining features are further selected by the grn method with 474 different parameters. The Svm group has 4 methods leveraging support vector machines with feature 475 selection methods "scad", "L1", "ElasticNet" and "scad+L2". The GSEA group has 10 methods based on gene set enrichment analysis. The features are chosen with either FDR (False discovery rate) $<0.1$ or 
477 FDR $<0.25$. The features are ordered by FDR value within both feature groups and selected by choosing 478 the lowest $10 \%$, lowest $20 \%$, lowest $50 \%$, or below mean threshold. The remaining features are filtered 479 by the cor and the grn3 method. The DE-analysis group has 15 methods and is picking features based on 480 differential gene expression. The methods pick features with the combination of $P$ value $(P<0.05$ or $P$ $481<0.01)$, fold change $(\operatorname{logFc}>0$ or $\operatorname{logFc}<0$ or both), and different numbers of features $(1000,500$, or 100 482 features with the lowest $P$ ).

483 The third step was feature reduction (Supplementary Figure 1). Seven methods were used: no (no 484 feature reduction), $\mathrm{WGCNA}^{61}$, logisticFwd, SIS $^{62}$, principal component regression (PCR) ${ }^{63}$, partial least 485 squares regression (PLSR) ${ }^{64}$, canonical powered partial least squares (CPPLS) ${ }^{64}$. The WGCNA, weighted correlation network analysis, calculates modules of co-expressed genes ${ }^{61}$, and we select hub genes from 487 each module. The LogisticFwd method deploys stepwise logistic regression with the forward method to 488 select features. The SIS ${ }^{62}$ method returns the features selected by the SIS R package, which implements 489 the "Iterative Sure Independence Screening" for selecting variants. PCR regresses on a subset of principal 490 components for dimensionality reduction, while PLSR finds a dimensionality-reducing linear regression 491 model by projecting the variables to a new space, and CPPLS allows for discrete and continuous responses 492 in the PLS model. After three steps, up to 1320 gene routes were created that can be used in the 493 classification step.

494 The classification step exploited 12 classifiers (Supplementary Figure 1), including reg (linear model), $495 \operatorname{logReg}^{65}$ (logistic regression), $\operatorname{lda}^{65}$ (Linear Discriminant Analysis), qda ${ }^{65}$ (Quadratic Discriminant 496 Analysis), ridgeReg66 (GLM with ridge regularization), lassoReg66 (GLM with lasso regularization), 497 ridgeLogReg ${ }^{66}$ (logistic regression with ridge regularization), lassoLogReg ${ }^{66}$ (logistic regression with 498 lasso regularization), elasticNetLogReg ${ }^{66}$ (logistic regression with elastic net regularization), boosting ${ }^{67}$ 499 (Generalized Boosted Regression Modeling with Bernoulli distribution), randomForest ${ }^{68}$ (random forest) 
500 and bagging ${ }^{68}$ (random forests with bagging to reduce the complexity). After training a classifier, the

501 diagnostic ability was evaluated by AUC-PR (precision-recall) curve and AUC-ROC (Receiver operating 502 characteristic) curve $\mathrm{e}^{44,69}$.

503 After five iterations, the averaged value of AUC area under precision $=0.95$ and precision=0.85 was 504 used to compare the feature route and classifier performance. $1822(11.5 \%)$ models with classification 505 AUC-ROC of above 0.8 were found.

506

\section{Label permutation data}

508 To generate the randomized background, we shuffled the diagnostic label of the Discovery dataset and 509 randomly separated the data into training/validation segments $(85 \% / 15 \%)$. Then we perform the 5-fold 510 cross validation on the permuted dataset.

\section{Random forest model created the baseline AUC-ROC}

513 Used 175 Discovery and 65 Replication dataset. Data are prepared by randomly splitting the Independent 514 Replication dataset into a separate validation and test set. The same Discovery dataset is used for training. 515 Using the Discovery set, we perform feature selection for the top $\boldsymbol{q}$ variables, as scored by the chi-squared 516 statistic. From a grid, $\boldsymbol{q}$ is chosen based on which grid value yields the highest mean AUC after 100 random 517 forest evaluations on the validation set. Additionally, we average the feature importance rankings over the 518100 iterations to provide a more comprehensive view of important genes. With the selected $\boldsymbol{q}$ value, we 519 train a random forest classifier and evaluate on the held-out test set. 
medRxiv preprint doi: https://doi.org/10.1101/2021.07.08.21260225; this version posted July 9, 2021. The copyright holder for this preprint (which was not certified by peer review) is the author/funder, who has granted medRxiv a license to display the preprint in perpetuity.

It is made available under a CC-BY-NC-ND 4.0 International license .

522 The 12-classifier mean AUC-ROC scores of 1320 routes are calculated and set as x-axis. 12

523 classifiers'AUC-ROC scores are visualized as y-axis (Supplementary Figure 3C and D). Then, the 12-

524 classifiers mean AUC-ROC scores of 1320 routes are binned into 10 quantiles and the variance of the $12-$

525 classifiers AUC-ROC score for each route are calculated and presented as boxplot (Supplementary Figure

$5263 \mathrm{E}$ and $\mathrm{F})$.

527

528 Reproducibility on Longitudinal dataset

529 Models were trained on the Discovery dataset (175 subjects) and then tested on the Longitudinal dataset

530 (Supplementary Figure 2). The Longitudinal dataset contained 18 ASD and 15 TD toddlers in the

531 Discovery set. 1656 (90.8\%) models showed AUC-ROC of $>0.8$ in the Longitudinal dataset.

532

533 Validation of Independent Replication dataset

534 The independent Replication dataset consisted of 89 subjects (34 ASD, 31 TD, and 24 LD). To test the 535 sensitivity, the models were trained on the Discovery dataset (175 subjects together) and then tested on 536 the dataset with 34 ASD and 31 TD. The AUC-ROC values were used to evaluate the performance of the 537 selected 1822 high-performing models. 1076 models (59.0\%) had an AUC-ROC value greater than 0.75 538 in the Replication dataset

Bayesian model averaging to create a single transcriptomic ensemble classifier from the 1822 models

541 After training on the Discovery dataset, the 1076 models that also had AUC-ROC value $>0.8$ on the

542 Replication dataset were selected. The ensemble score was the sum of weighted predictions of selected

543 models. The weight is the mathematical average of the square of (AUC-ROC value minus 0.7). In a

544 model selection, we use training data D to select a good model M (according to some score) to use in 
545 predicting a targeted outcome $\mathrm{T}$ of interest based on patient features $\mathrm{X}$, namely, $\mathrm{P}(\mathrm{T} \mid \mathrm{X}, \mathrm{M})$. BMA is

546 based on the notion of averaging over a set of possible models and weighting the prediction of each

547 model according to its probability given training data $\mathrm{D}$, as shown in equations.

$$
\text { - } p(T \mid X)=\sum_{m_{i}} p\left(M_{i} \mid X\right) p\left(T \mid X, M_{i}\right)
$$

$549 \mathrm{M}$ is the model, $\mathrm{T}$ is the prediction and $\mathrm{X}$ is the data.

$$
\text { - } \mathrm{p}\left(\mathrm{M}_{\mathrm{i}} \mid \mathrm{X}\right)=\frac{\text { AUCROC }_{\mathrm{i}}-0.7}{\sum_{\mathrm{j}}\left(\mathrm{AUCROC}_{\mathrm{i}}-0.7\right)}
$$

551 The ensemble scores of the independent dataset are calculated based on the same model built. The scores 552 are then rescaled to 0 and 1.

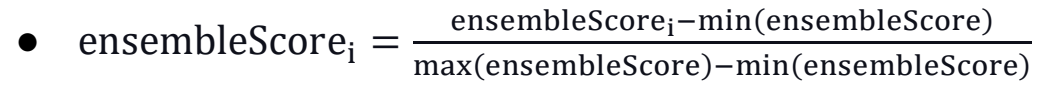

\section{The gene set similarity score between routes}

556 We compared the first five hundred genes that existed in the 191 routes (Supplementary Table 2). In

557 order to retrieve the genes from dimension deduction methods such pcr, cppls and plsr, we calculated the 558 feature weights and the gene weights in each feature. Then, we ranked the genes with the weights they 559 contribute to all features and selected the genes that cumulated to $66 \%$ of total weights.

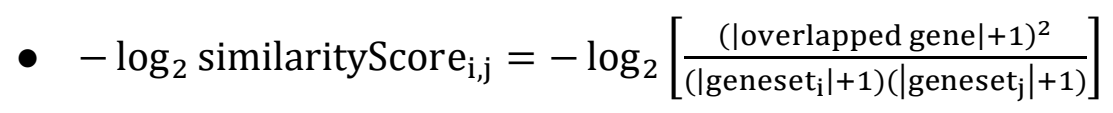

561 In the Supplementary Figure 5 and 6, the distance of the routes gene similarity is presented as

$562-\log _{2}$ similarityScore $e_{i, j}$

\section{Calculating the biological enrichment of DE genes between above-mean ASD and TD}

565 After generating the prediction score by the ensemble classifier, we stratified ASD toddlers in the

566 Discovery dataset into those with above and below the Discovery dataset's ensemble classifier mean score 
of 0.714 . Those above this threshold were considered "above-the-mean" and below 0.714 are considered

568

569

570

571

572

573

574

575

576

577

578

579

580

581

582

583

584

585

586

587

588

589

"below-the-mean". Then we conducted differential expression (DE) analysis on the above-the-mean group $(\mathrm{n}=59)$ subjects and TD $(\mathrm{n}=82)$ subjects. Then we conducted differential expression (DE) analysis on the below-the-mean group $(n=34)$ and TD $(n=82)$ subjects. The limma package ${ }^{60,70}$ was then applied on quantile-normalized data for differential expression analysis in which moderated t-statistics were calculated by robust empirical Bayes methods. We use adj.p.value $<0.01$ and $\log \mathrm{FC}>0.1$ to select genes and generate the volcano plot. The Gene Ontology (GO) enrichment was conducted using g:Profiler ${ }^{48}$ (https://biit.cs.ut.ee/gprofiler/gost) with 12695 protein-coding genes (12695/14132 gene features) as background (g:Profiler, advanced option/statistical domain scope: Custom; custom over annotated genes).

We only checked the "GO biological process" and KEGG terms of size 15-1500 in the biological process. The threshold is "Significance threshold: B-H FDR $<0.1$ ". Then the terms are clustered with REVIGO ${ }^{71}$, ordered with $P$ value (http://revigo.irb.hr/). The connections across terms are visualized by the Cytoscape 3.8. $2^{72}$

\section{Clinical and prenatal characteristics associated with ASD classifier scores}

To examine clinical and prenatal characteristics associated with ensemble classifier scores, we stratified 127 ASD toddlers in the Discovery and independent Replication datasets into those with above and below the score of 0.714 (again as the easy and the hard group). The t-test is used to compare the diagnostic and psychometric scores between ASD toddlers among two groups (Table 3). Among 127 subjects, 124 subjects have complete prenatal records. The t-test was used to compare the diagnostic and psychometric scores between ASD toddlers among two groups (Table 3). Among 127 subjects, 124 subjects had complete prenatal records. The fisher.t.test was used to compare the prenatal risk factors across easy, hard and TD groups. Next, we stratified ASD toddlers based on the group means of CoSo Total symptom 
medRxiv preprint doi: https://doi.org/10.1101/2021.07.08.21260225; this version posted July 9, 2021. The copyright holder for this preprint (which was not certified by peer review) is the author/funder, who has granted medRxiv a license to display the preprint in perpetuity.

It is made available under a CC-BY-NC-ND 4.0 International license.

590 severity and Mullen cognitive scores. As shown in Supplementary Table 2, these clinical stratifiers also

591 did not differentiate ASD toddlers with higher or lower ensemble classifier scores. Thus, the ensemble

592 classifier was equally effective as a diagnostic tool across a considerable range of early-age ASD

593 diagnostic symptoms and cognitive presentation.

594 We performed analogous stratifications within the TD Discovery group and found no ADOS or Mullen 595 differences between higher or lower than the TD mean ensemble classifier score, nor differences in the 596 ensemble scores of TD toddlers with high vs lower diagnostic and psychometric scores (Supplementary 597 Table 2).

598

599 Geo Fixation (non-social) characteristics associated with ASD classifier scores

600 After we have the ensemble score, we tested the ensemble model on 132/175 of the subjects found in 601 the Discovery dataset that have high quality Geo Fixation (non-social) data and 41/65 of the subjects found 602 in the independent Replication dataset. The rule is

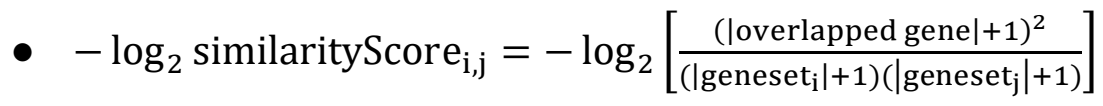

604

- ensembleScore $_{i}=1$ if geo-fixation percentage $>69 \%$, else ensembleScore ${ }_{i}$

605

606

607 
medRxiv preprint doi: https://doi.org/10.1101/2021.07.08.21260225; this version posted July 9, 2021. The copyright holder for this preprint (which was not certified by peer review) is the author/funder, who has granted medRxiv a license to display the preprint in perpetuity.

It is made available under a CC-BY-NC-ND 4.0 International license .

\section{Reference}

1. Courchesne E, Gazestani VH, Lewis NE. Prenatal origins of ASD: The when, what, and how of ASD development. Trends Neurosci. 2020;43(5):326-342.

2. Courchesne E, Pramparo T, Gazestani VH, Lombardo MV, Pierce K, Lewis NE. The ASD Living Biology: from cell proliferation to clinical phenotype. Mol Psychiatry. 2019;24(1):88-107.

3. Gazestani V, Chiang AWT, Courchesne E, Lewis NE. Autism genetics perturb prenatal neurodevelopment through a hierarchy of broadly-expressed and brain-specific genes. bioRxiv. Published online 2020.

4. Courchesne E, Mouton PR, Calhoun ME, et al. Neuron number and size in prefrontal cortex of children with autism. JAMA. 2011;306(18):2001-2010.

5. Marchetto MC, Belinson $\mathrm{H}$, Tian Y, et al. Altered proliferation and networks in neural cells derived from idiopathic autistic individuals. Mol Psychiatry. 2017;22(6):820-835.

6. Courchesne E, Pierce K. Why the frontal cortex in autism might be talking only to itself: local overconnectivity but long-distance disconnection. Curr Opin Neurobiol. 2005;15(2):225-230.

7. Willsey AJ, Sanders SJ, Li M, et al. Coexpression networks implicate human midfetal deep cortical projection neurons in the pathogenesis of autism. Cell. 2013;155(5):997-1007.

8. Courchesne E, Pierce K, Schumann CM, et al. Mapping early brain development in autism. Neuron. 2007;56(2):399-413.

9. Stoner R, Chow ML, Boyle MP, et al. Patches of Disorganization in the Neocortex of Children with Autism. New England Journal of Medicine. 2014;370(13):1209-1219. doi:10.1056/nejmoa1307491

10. Parikshak NN, Luo R, Zhang A, et al. Integrative Functional Genomic Analyses Implicate Specific Molecular Pathways and Circuits in Autism. Cell. 2013;155(5):1008-1021. doi:10.1016/j.cell.2013.10.031

11. Packer A. Neocortical neurogenesis and the etiology of autism spectrum disorder. Neurosci Biobehav Rev. 2016;64:185-195.

12. Kaushik G, Zarbalis KS. Prenatal Neurogenesis in Autism Spectrum Disorders. Frontiers in Chemistry. 2016;4. doi:10.3389/fchem.2016.00012

13. Krishnan A, Zhang R, Yao V, et al. Genome-wide prediction and functional characterization of the genetic basis of autism spectrum disorder. Nat Neurosci. 2016;19(11):1454-1462.

14. Donovan APA, Basson MA. The neuroanatomy of autism - a developmental perspective. $J$ Anat. 2017;230(1):4-15.

15. Grove J, Ripke S, Als TD, et al. Identification of common genetic risk variants for autism spectrum disorder. Nat Genet. 2019;51(3):431-444.

16. Satterstrom FK, Kosmicki JA, Wang J, et al. Large-Scale Exome Sequencing Study Implicates Both Developmental and Functional Changes in the Neurobiology of Autism. Cell. 2020;180(3):568-584.e23.

17. Bai D, Yip BHK, Windham GC, et al. Association of Genetic and Environmental Factors With Autism in a 5-Country Cohort. JAMA Psychiatry. 2019;76(10):1035-1043. 
643

644

645

646

647

648

649

650

651

652

653

654

655

656

657

658

659

660

661

662

663

664

665

666

667

668

669

670

671

672

673

674

675

676

677

678

679

680

18. Bal VH, Kim S-H, Fok M, Lord C. Autism spectrum disorder symptoms from ages 2 to 19 years: Implications for diagnosing adolescents and young adults. Autism Res. 2019;12(1):89-99.

19. Bacon EC, Courchesne E, Barnes CC, et al. Rethinking the idea of late autism spectrum disorder onset. Dev Psychopathol. 2018;30(2):553-569.

20. Bacon EC, Osuna S, Courchesne E, Pierce K. Naturalistic language sampling to characterize the language abilities of 3-year-olds with autism spectrum disorder. Autism. 2019;23(3):699-712.

21. Autism and Developmental Disabilities Monitoring Network Surveillance Year 2006 Principal Investigators, Centers for Disease Control and Prevention (CDC). Prevalence of autism spectrum disorders - Autism and Developmental Disabilities Monitoring Network, United States, 2006. MMWR Surveill Summ. 2009;58(10):1-20.

22. Baio J, Wiggins L, Christensen DL, et al. Prevalence of Autism Spectrum Disorder Among Children Aged 8 Years - Autism and Developmental Disabilities Monitoring Network, 11 Sites, United States, 2014. MMWR Surveill Summ. 2018;67(6):1-23.

23. Christensen DL, Braun KVN, Baio J, et al. Prevalence and Characteristics of Autism Spectrum Disorder Among Children Aged 8 Years - Autism and Developmental Disabilities Monitoring Network, 11 Sites, United States, 2012. MMWR Surveill Summ. 2018;65(13):1-23.

24. Maenner MJ, Shaw KA, Baio J, et al. Prevalence of Autism Spectrum Disorder Among Children Aged 8 Years - Autism and Developmental Disabilities Monitoring Network, 11 Sites, United States, 2016. MMWR Surveill Summ. 2020;69(4):1-12.

25. Lombardo MV, Lai M-C, Baron-Cohen S. Big data approaches to decomposing heterogeneity across the autism spectrum. Mol Psychiatry. 2019;24(10):1435-1450.

26. Robinson EB, St Pourcain B, Anttila V, et al. Genetic risk for autism spectrum disorders and neuropsychiatric variation in the general population. Nat Genet. 2016;48(5):552-555.

27. Ahn K, An SS, Shugart YY, Rapoport JL. Common polygenic variation and risk for childhood-onset schizophrenia. Mol Psychiatry. 2016;21(1):94-96.

28. Clarke T-K, Lupton MK, Fernandez-Pujals AM, et al. Common polygenic risk for autism spectrum disorder (ASD) is associated with cognitive ability in the general population. Mol Psychiatry. 2016;21(3):419-425.

29. Pramparo T, Lombardo MV, Campbell K, et al. Cell cycle networks link gene expression dysregulation, mutation, and brain maldevelopment in autistic toddlers. Mol Syst Biol. 2015;11(12):841.

30. Pramparo T, Pierce K, Lombardo MV, et al. Prediction of autism by translation and immune/inflammation coexpressed genes in toddlers from pediatric community practices. JAMA Psychiatry. 2015;72(4):386-394.

31. Ch'ng C, Kwok W, Rogic S, Pavlidis P. Meta-Analysis of Gene Expression in Autism Spectrum Disorder. Autism Res. 2015;8(5):593-608.

32. Diaz-Beltran L, Esteban FJ, Wall DP. A common molecular signature in ASD gene expression: following Root 66 to autism. Transl Psychiatry. 2016;6:e705.

33. Tylee DS, Hess JL, Quinn TP, et al. Blood transcriptomic comparison of individuals with and without autism spectrum disorder: A combined-samples mega-analysis. Am J Med Genet B Neuropsychiatr Genet. 2017;174(3):181-201. 
medRxiv preprint doi: https://doi.org/10.1101/2021.07.08.21260225; this version posted July 9, 2021. The copyright holder for this preprint (which was not certified by peer review) is the author/funder, who has granted medRxiv a license to display the preprint in perpetuity. It is made available under a CC-BY-NC-ND 4.0 International license .

681

682

683

684

685

686

687

688

689

690

691

692

693

694

695

696

697

698

699

700

701

702

703

704

705

706

707

708

709

710

711

712

713

714

715

716

34. He Y, Zhou Y, Ma W, Wang J. An integrated transcriptomic analysis of autism spectrum disorder. Sci Rep. 2019;9(1):11818.

35. Lee SC, Quinn TP, Lai J, et al. Solving for X: Evidence for sex-specific autism biomarkers across multiple transcriptomic studies. Am J Med Genet B Neuropsychiatr Genet. 2019;180(6):377-389.

36. Kong SW, Collins CD, Shimizu-Motohashi Y, et al. Characteristics and predictive value of blood transcriptome signature in males with autism spectrum disorders. PLoS One. 2012;7(12):e49475.

37. Gregg JP, Lit L, Baron CA, et al. Gene expression changes in children with autism. Genomics. 2008;91(1):22-29.

38. Enstrom AM, Lit L, Onore CE, et al. Altered gene expression and function of peripheral blood natural killer cells in children with autism. Brain Behav Immun. 2009;23(1):124-133.

39. Ansel A, Rosenzweig JP, Zisman PD, Melamed M, Gesundheit B. Variation in Gene Expression in Autism Spectrum Disorders: An Extensive Review of Transcriptomic Studies. Front Neurosci. 2016;10:601.

40. Hyde KK, Novack MN, LaHaye N, et al. Applications of Supervised Machine Learning in Autism Spectrum Disorder Research: a Review. Review Journal of Autism and Developmental Disorders. 2019;6(2):128-146.

41. Hewitson L, Mathews JA, Devlin M, Schutte C, Lee J, German DC. Blood biomarker discovery for autism spectrum disorder: A proteomic analysis. PLoS One. 2021;16(2):e0246581.

42. Gazestani VH, Pramparo T, Nalabolu S, et al. A perturbed gene network containing PI3K-AKT, RAS-ERK and WNT- $\beta$-catenin pathways in leukocytes is linked to ASD genetics and symptom severity. Nat Neurosci. 2019;22(10):1624-1634.

43. Pierce K, Gazestani VH, Bacon E, et al. Evaluation of the Diagnostic Stability of the Early Autism Spectrum Disorder Phenotype in the General Population Starting at 12 Months. JAMA Pediatr. 2019;173(6):578-587.

44. Robin X, Turck N, Hainard A, et al. pROC: an open-source package for R and S+ to analyze and compare ROC curves. BMC Bioinformatics. 2011;12:77.

45. Pierce K, Conant D, Hazin R, Stoner R, Desmond J. Preference for geometric patterns early in life as a risk factor for autism. Arch Gen Psychiatry. 2011;68(1):101-109.

46. Moore A, Wozniak M, Yousef A, et al. The geometric preference subtype in ASD: identifying a consistent, early-emerging phenomenon through eye tracking. Mol Autism. 2018;9:19.

47. Pierce K, Marinero S, Hazin R, McKenna B, Barnes CC, Malige A. Eye Tracking Reveals Abnormal Visual Preference for Geometric Images as an Early Biomarker of an Autism Spectrum Disorder Subtype Associated With Increased Symptom Severity. Biol Psychiatry. 2016;79(8):657-666.

48. Raudvere U, Kolberg L, Kuzmin I, et al. g:Profiler: a web server for functional enrichment analysis and conversions of gene lists (2019 update). Nucleic Acids Res. 2019;47(W1):W191-W198.

49. Kanehisa M, Goto S. KEGG: kyoto encyclopedia of genes and genomes. Nucleic Acids Res. 2000;28(1):2730.

50. Lombardo MV, Eyler L, Pramparo T, et al. Atypical genomic patterning of the cerebral cortex in autism with poor early language outcome. doi:10.1101/2020.08.18.253443 
51. Lombardo MV, Pramparo T, Gazestani V, et al. Large-scale associations between the leukocyte transcriptome and BOLD responses to speech differ in autism early language outcome subtypes. Nat Neurosci. 2018;21(12):1680-1688.

52. Lombardo MV, Busuoli EM, Schreibman L, et al. Pre-treatment clinical behavioral and blood leukocyte gene expression patterns predict rate of change in response to early intervention in autism. medRxiv. Published online 2020. https://www.medrxiv.org/content/10.1101/2020.12.21.20248674v1.abstract

53. Lombardo MV, Eyler L, Pramparo T, Gazestani VH. Atypical genomic cortical patterning in autism with poor early language outcome. bioRxiv. Published online 2021.

https://www.biorxiv.org/content/10.1101/2020.08.18.253443v3.abstract

54. Du P, Kibbe WA, Lin SM. lumi: a pipeline for processing Illumina microarray. Bioinformatics. 2008;24(13):1547-1548. doi:10.1093/bioinformatics/btn224

55. Ardlie KG, Deluca DS, Segre AV, et al. The Genotype-Tissue Expression (GTEx) pilot analysis: Multitissue gene regulation in humans. Science. 2015;348(6235):648-660.

56. Meyer PE, Lafitte F, Bontempi G. minet: A R/Bioconductor package for inferring large transcriptional networks using mutual information. BMC Bioinformatics. 2008;9:461.

57. Antonio Pedro Duarte Silva <psilva @ porto.ucp.pt>. SelectV: Variable selection for high-dimensional supervised... In HiDimDA: High dimensional Discriminant Analysis. Published October 19, 2015. Accessed May 21, 2021. https://rdrr.io/cran/HiDimDA/man/SelectV.html

58. penalizedSVM: Feature Selection SVM using Penalty Functions. Accessed June 29, 2021. https://cran.rproject.org/web/packages/penalizedSVM/index.html

59. Subramanian A, Tamayo P, Mootha VK, et al. Gene set enrichment analysis: a knowledge-based approach for interpreting genome-wide expression profiles. Proc Natl Acad Sci U S A. 2005;102(43):15545-15550.

60. Ritchie ME, Phipson B, Wu D, et al. limma powers differential expression analyses for RNA-sequencing and microarray studies. Nucleic Acids Research. 2015;43(7):e47-e47. doi:10.1093/nar/gkv007

61. Langfelder P, Horvath S. WGCNA: an R package for weighted correlation network analysis. $B M C$ Bioinformatics. 2008;9:559.

62. Saldana DF, Feng Y. SIS: An R Package for Sure Independence Screening in Ultrahigh-Dimensional Statistical Models. Journal of Statistical Software, Articles. 2018;83(2):1-25.

63. Mevik B-H, Wehrens R. Introduction to the pls Package. Help Section of The "Pls" Package of R Studio Software; R Foundation for Statistical Computing: Vienna, Austria. Published online 2015:1-23.

64. Wehrens R, Mevik B-H. The pls package: principal component and partial least squares regression in R. Published online 2007. https://repository.ubn.ru.nl/bitstream/handle/2066/36604/36604.pdf

65. Ripley BD. Modern Applied Statistics with S. springer; 2002.

66. Friedman J, Hastie T, Tibshirani R. Regularization paths for generalized linear models via coordinate descent. J Stat Softw. 2010;33(1):1-22.

67. Ridgeway G. Generalized Boosted Models: A guide to the gbm package. Update. 2007;1(1):2007. 
medRxiv preprint doi: https://doi.org/10.1101/2021.07.08.21260225; this version posted July 9, 2021. The copyright holder for this preprint (which was not certified by peer review) is the author/funder, who has granted medRxiv a license to display the preprint in perpetuity. It is made available under a CC-BY-NC-ND 4.0 International license.

753 68. Liaw A, Wiener M, Others. Classification and regression by randomForest. $R$ news. 2002;2(3):18-22.

754 69. Grau J, Grosse I, Keilwagen J. PRROC: computing and visualizing precision-recall and receiver operating characteristic curves in R. Bioinformatics. 2015;31(15):2595-2597.

70. Smyth GK. limma: Linear Models for Microarray Data. In: Gentleman R, Carey VJ, Huber W, Irizarry RA, Dudoit S, eds. Bioinformatics and Computational Biology Solutions Using R and Bioconductor. Springer New York; 2005:397-420.

71. Supek F, Bošnjak M, Škunca N, Šmuc T. REVIGO summarizes and visualizes long lists of gene ontology terms. PLoS One. 2011;6(7):e21800.

72. Su G, Morris JH, Demchak B, Bader GD. Biological network exploration with Cytoscape 3. Curr Protoc Bioinformatics. 2014;47:8.13.1-24. 
medRxiv preprint doi: https://doi.org/10.1101/2021.07.08.21260225; this version posted July 9, 2021. The copyright holder for this preprint (which was not certified by peer review) is the author/funder, who has granted medRxiv a license to display the preprint in perpetuity.

It is made available under a CC-BY-NC-ND 4.0 International license .

Figure 1. A classification platform was developed to robustly identify the biomarkers for the early diagnosis of ASD.

A

Feature engineering

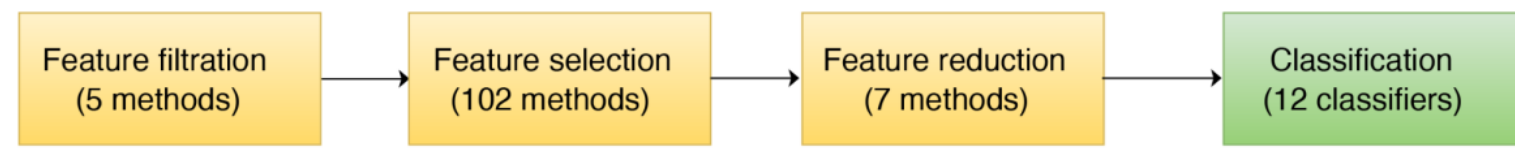

B AUC-ROC score

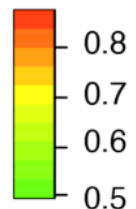

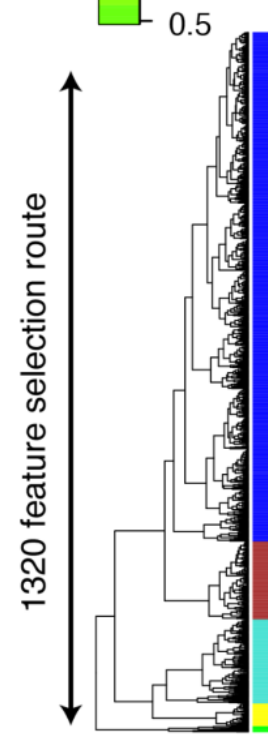

\section{AUC-ROC scores for all datasets}

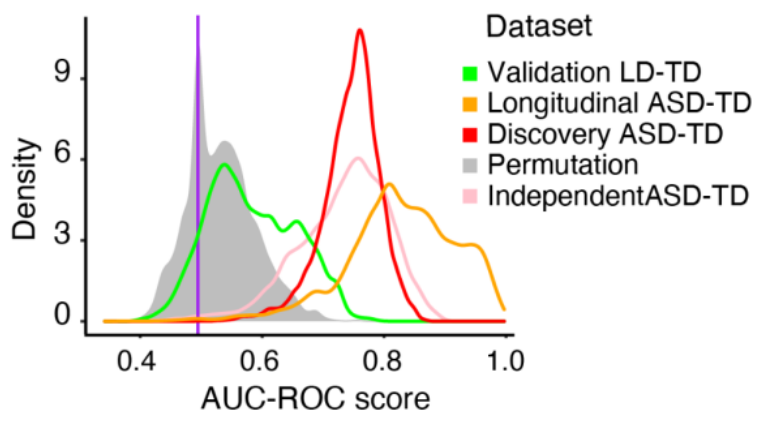

D Top 5\% permutation AUC-ROC scores

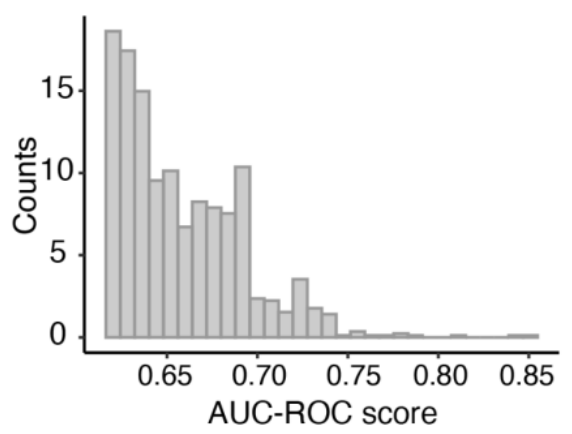

767 A. Our platform performs 3570 feature engineering methods, followed by 12 different classification approaches on the input datasets. B. AUC-ROC values were computed for each feature selection route and classification method on the Discovery dataset. The AUC-ROC values are based on the average model performance across 5 iterations, with $20 \%$ of samples being held out each time. C. 15,840 models were

771 further tested on an independent Replication dataset and Longitudinal samples from the Discovery dataset, and on average performed well. Evaluation of the models on a dataset of language delay subjects showed the specificity of the signals to ASD subjects. Permuting the sample labels (i.e., ASD and TD) further supported the validity of the signal. D. Top 5\% AUC-ROC score of permutation data. 
medRxiv preprint doi: https://doi.org/10.1101/2021.07.08.21260225; this version posted July 9, 2021. The copyright holder for this preprint (which was not certified by peer review) is the author/funder, who has granted medRxiv a license to display the preprint in perpetuity.

It is made available under a CC-BY-NC-ND 4.0 International license .

Figure 2. Blood transcriptome ASD subtypes identified by our classification platform.

A

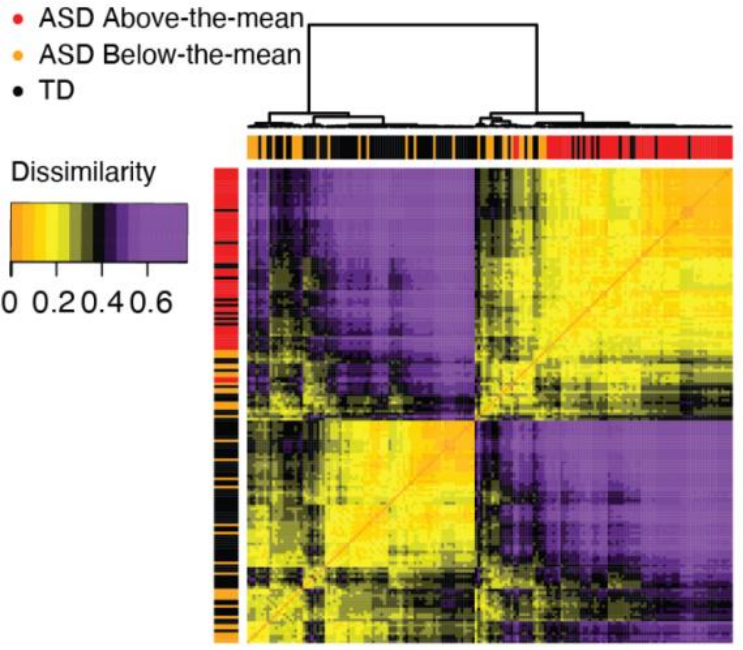

Replication Dataset

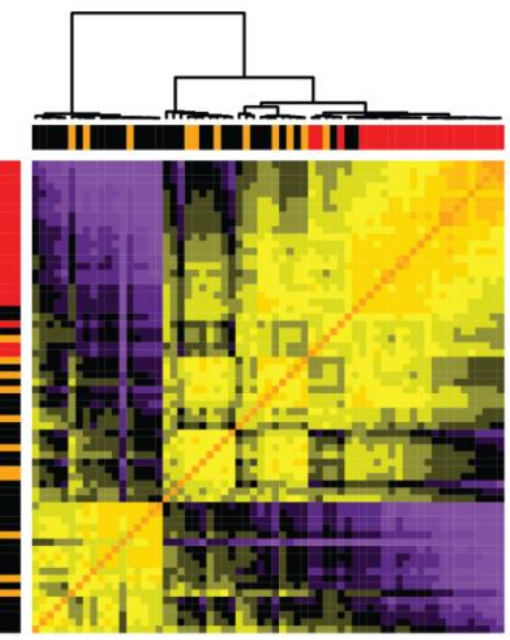

B

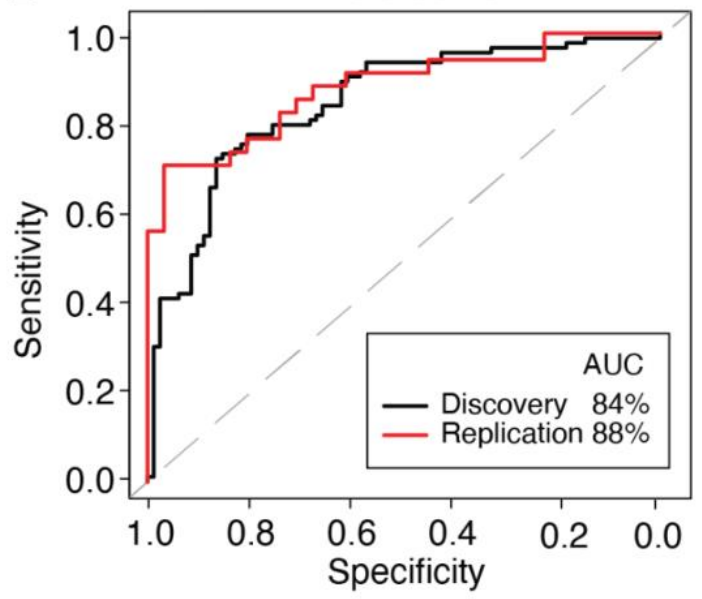

D

DE result for 2764 feature genes in the Discovery Dataset

Above-the-mean subgroup Below-the-mean

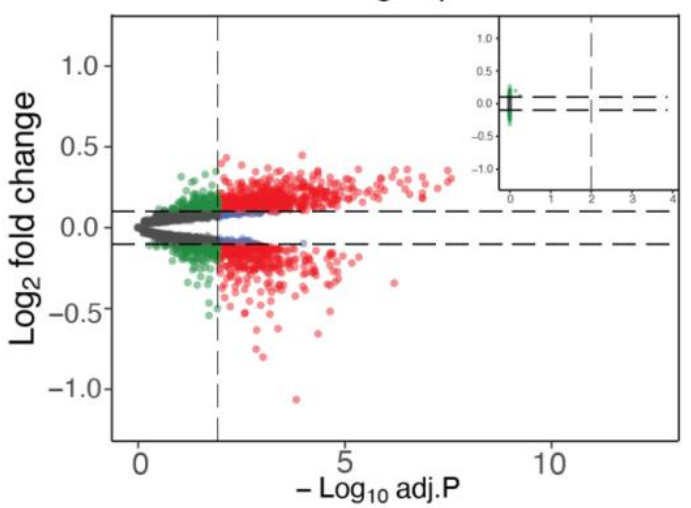

NS $\log _{2}$ FC $\bigcirc$-value $\log _{2}$ fold change cutoff 0.1
C The ensemble score over two datasets

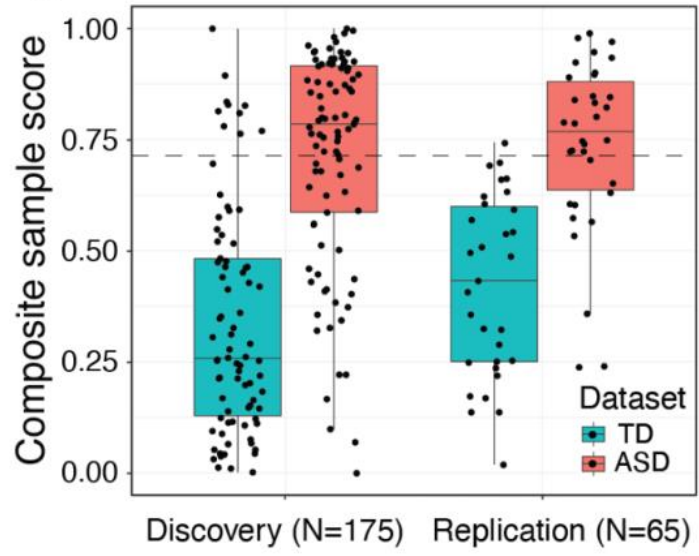

E DE result for 2764 feature genes in the Replication Dataset

Above-the-mean subgroup Below-the-mean

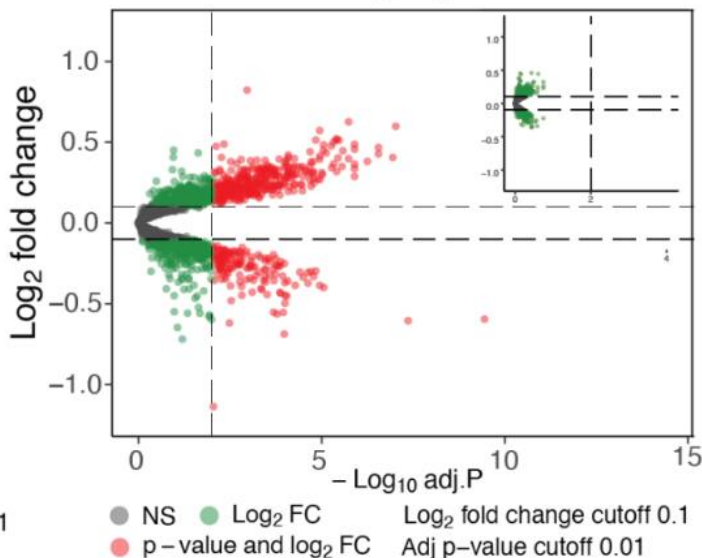


777 A. ASD and TD subjects show distinct classification patterns. The classification scores for each subject

778 in the evaluation set is based on their score in the held-out sample. The red, orange and black bars on the

779 sides represent above-the-mean ASD, below-the-mean ASD and TD subjects respectively. The orange 780 and purple colors represent the gradient of dissimilarity between subjects based on their classification

781 scores. B. The AUC-ROC results on the ensemble classification model generated by the Baysician model

782 averaging approach. C. Subject ensemble scores based on the ensemble model. The quantile information

783 of the main TD group is Min=0.0029, Q1=0.1337, Median=0.2568, Q3=0.4866, Max=0.9996; main ASD

784 group is Min=0, Q1=0.576, Median=0.7859, Q3=0.9161, Max=1; Replication TD group is Min=0.0168, $785 \mathrm{Q} 1=0.2454$, Median=0.4196, Q3=0.5862, Max=0.7349; Replication ASD group is Min=0.238, Q1=0.628,

786 Median=0.762, Q3=0.879, Max=0.990. D. and E. The differential expression analysis of 2764 protein 787 coding feature genes. The volcano plots showed the adjusted $P$ value (cutoff $=0.01$ ) vs $\operatorname{logFold}$ changes 788 (cutoff $=0.1$ ) of genes in the above-the-mean to TD subjects and below-the-mean subjects to TD subjects 789 in Discovery dataset and Replication dataset. 
Table 1. Summary of the Subjects

\begin{tabular}{|l|c|c|c|}
\hline Dateset & ASD & TD & LD \\
\hline Discovery & 93 & 82 & 0 \\
\hline Independent Replication & 34 & 31 & 24 \\
\hline Longitudinal & 18 & 15 & 0 \\
\hline
\end{tabular}

ASD: autism spectrum disorder; TD: typical development; LD: language delayed

Table 2. Distribution of Prenatal Events Among the Three Groups

\begin{tabular}{|c|c|c|c|}
\hline Severe prenatal events & $\begin{array}{c}\text { ASD } \\
\text { Above-the-mean }\end{array}$ & ASD Below-the-mean & $\mathrm{TD}$ \\
\hline Total subjects (n) & 79 & 45 & 107 \\
\hline $\begin{array}{l}\text { Hospitalizations during } \\
\text { pregnancy }\end{array}$ & 5 & 5 & 6 \\
\hline Surgery during pregnancy & 1 & 3 & 4 \\
\hline $\begin{array}{l}\text { Confinement to bed during } \\
\text { pregnancy }\end{array}$ & 8 & 8 & 9 \\
\hline $\begin{array}{l}\text { General Anesthesia during } \\
\text { delivery }\end{array}$ & 10 & 11 & 10 \\
\hline Total (\%) & $19(24.1)$ & $20(44.4)$ & $24(22.4)$ \\
\hline \multicolumn{4}{|l|}{ Negative control events } \\
\hline Nausea & 4 & 2 & 12 \\
\hline Morning sickness & 42 & 18 & 64 \\
\hline Swelling & 23 & 10 & 26 \\
\hline Total $(\%)$ & $52(65.8)$ & $24(53.3)$ & $70(65.4)$ \\
\hline
\end{tabular}

792 ASD above-the-mean: autism spectrum disorder with ensemble score over 0.714; ASD below-the-mean:

793 below 0.714; TD: typical development. 
medRxiv preprint doi: https://doi.org/10.1101/2021.07.08.21260225; this version posted July 9, 2021. The copyright holder for this preprint (which was not certified by peer review) is the author/funder, who has granted medRxiv a license to display the preprint in perpetuity.

It is made available under a CC-BY-NC-ND 4.0 International license .

Table 3. Statistical Differences between the Three Groups

\begin{tabular}{|l|c|c|}
\hline & Odds ratio & $P$ value \\
\hline ASD above-the-mean vs. TD & 0.914 & 0.861 \\
\hline ASD below-the-mean vs. TD & 2.746 & 0.0102 \\
\hline ASD hard- vs. above-the-mean & 2.506 & 0.027 \\
\hline
\end{tabular}

$P$ value is calculated by the two-sided Fisher's exact test 